\title{
SUPPLEMENTARY DIFFERENCE SETS WITH SYMMETRY FOR HADAMARD MATRICES
}

\author{
DRAGOMIR Ž. ĐOKOVIĆ
}

Abstract. An overview of the known supplementary difference sets (SDSs) $\left(A_{i}\right), 1 \leqslant i \leqslant 4$, with parameters $\left(n ; k_{i} ; \lambda\right), k_{i}=\left|A_{i}\right|$, where each $A_{i}$ is either symmetric or skew and $\sum k_{i}=n+\lambda$ is given. Five new Williamson matrices over the elementary abelian groups of order $5^{2}, 3^{3}$ and $7^{2}$ are constructed. New examples of skew Hadamard matrices of order $4 n$ for $n=47,61,127$ are presented. The last of these is obtained from a $(127,57,76)$ difference family that we have constructed. An old non-published example of G-matrices of order 37 is also included.

Mathematics subject classification (2000): 05B20, 05B30.

Keywords and phrases: Supplementary difference sets, Hadamard matrices, Williamson matrices, GoethalsSeidel array.

\section{REFERENCES}

[1] L.D. Baumert And M. Hall JR., Hadamard matrices of the Williamson type, Math. Comput., 19 (1965), 442-447.

[2] D.Ž. ĐoKović, Williamson matrices of orders $4 \cdot 29$ and 4·31, J. Combin. Theory Ser. A, 59 (1992), 309-311.

[3] D.Ž. Đoković, Williamson matrices of order $4 n$ for $n=33,35,39$, Discrete Mathematics, 115 (1993), 267-271.

[4] D.Ž. Đoković, Six new orders for G-matrices and some new orthogonal designs, Journal of Combinatorics, Information \& System Sciences 20, Nos. 1-4, (1995), 1-7.

[5] D.Ž. ĐoKović, Note on Williamson matrices of orders 25 and 37, JCMCC, 18 (1995), 171-175.

[6] D.Ž. Đокоvić, Skew-Hadamard matrices of orders 188 and 388 exist, International Mathematical Forum, 3, 22 (2008), 1063-1068.

[7] S. Georgiou And C. Koukouvinos, On circulant G-matrices, JCMCC, 40 (2002), 205-225.

[8] S. Georgiou, C. Koukouvinos and J. Seberry, On circulant best matrices and their applications, Linear and Multilinear Algebra, 48 (2001), 263-274.

[9] S. Georgiou, C. Koukouvinos and S. Stylianou, On good matrices, skew Hadamard matrices and optimal designs, Computational Statistics \& Data Analysis, 41 (2002), 171-184.

[10] J.M. Goethals And J.J. Seidel, A skew Hadamard matrix of oder 36, J. Austral. Math. Soc., 11 (1970), 343-344.

[11] W.H. Holzmann, H. Kharaghani And B. Tayfeh-Rezaie, Williamson matrices up to order 59, Des. Codes Cryptogr., 46 (2008), 343-352.

[12] J. Horton, C. KOUKOUVINOS AND J. SEBERRY, A search for Hadamard matrices constructed from Williamson matrices, Bull. Inst. Combin. Appl., 35 (2002), 75-88.

[13] DAVID HUNT, see [17, p. 471].

[14] Y.J. IONIN AND H. KHARAGHANI, Balanced generalized weighing matrices and conference matrices, in Handbook of Combinatorial Designs, 2nd edition, Eds. C.J. Colbourn and J.H. Dinitz, Chapman \& Hall, Boca Raton/London/New York, 2007, pp. 306-313.

[15] C. Koukouvinos and S. Stylianou, On skew-Hadamard matrices, Discrete Mathematics, 308 (2008), 2723-2731.

[16] K. SAWADE, Hadamard matrices of order 100 and 108, Bull. Nagoya Inst. Tech., 29 (1977), 147-153. 
[17] J. Seberry Wallis, Hadamard matrices, Part 4, 273-489. In Combinatorics: Room Squares, SumFree Sets, Hadamard Matrices, W.D. Wallis, A.P. Street, J.S. Wallis, eds., Lecture Notes in Mathematics No. 292, Springer, 1972.

[18] www.uow.edu.au/ jennie/WILLIAMSON/turyn...

[19] J. SEBERRY AND M. YAMADA, Hadamard matrices, sequences and block designs, in Contemporary Design Theory: A Collection of Surveys, Eds. J.H. Dinitz and D.R. Stinson, J. Wiley, New York, 1992, pp. 431-560.

[20] E. SPENCE, Skew-Hadamard matrices of order 2( $q+1)$, Discrete Mathematics, 18 (1977), 79-85.

[21] G. SzEKERES, A note on skew type orthogonal \pm 1 matrices, in A. Hajnal, L. Lovasz, V.T. Sos (Eds), Combinatorics, Colloquia Mathematica Societatis Janos Bolyai, No. 52. North Holland, Amsterdam, pp. $489-498$.

[22] R. TURYN, An infinite class of Williamson matrices, J. Combin. Theory Ser. A, 12 (1972), 319-321.

[23] RUDY VAN VLIET, The exhaustive search for Williamson matrices of order 45 and 51 is attributed to him, see e.g. [11].

[24] A.L. Whiteman, Hadamard matrices of Williamson type, J. Austral. Math. Soc. Ser. A, 21 (1976), $481-486$.

[25] M. XIA AND G. LiU, An infinite class of supplementary difference sets and Williamson matrices, J. Combin. Theory Ser. A, $\mathbf{5 8}$ (1991), 310-317.

[26] Q. XIANG, Difference families from lines and half lines, Europ. J. Combinatorics, 19 (1998), 395-400. 